\title{
Is Bisphenol A (BPA) a Threat to Children's Behavior?
}

\author{
Vicente Mustieles', Carmen Messerlian'², Iris Reina', Andrea Rodríguez-Carrillo', Nicolás Olea', and Mariana F. \\ Fernández ${ }^{1 *}$ \\ *'University of Granada, Center for Biomedical Research (CIBM); Biosanitary Research Institute of Granada (ibs.GRANADA), Spain; Consortium for Biomedical \\ Research in Epidemiology \& Public Health (CIBERESP), Spain. \\ ${ }^{2}$ Department of Environmental Health, Harvard T.H. Chan School of Public Health, Boston, MA, USA.
}

Article Info

\section{Article Notes}

Received: February 02, 2018

Accepted: March 05, 2018

\section{${ }^{*}$ Correspondence:}

Dr. Mariana F. Fernández Universidad de Granada. Instituto de Investigación Biosanitaria ibs. GRANADA, Spain; Tel.

Phone: +34 958241000 ext 20367

E-mail address: marieta@ugr.es

๑ 2018 Fernández MF. This article is distributed under the terms of the Creative Commons Attribution 4.0 International License.

\section{Keywords}

Bisphenol A

Bisphenol S

Bisphenol $F$

Behavior

Neurodevelopment

Endocrine Disruption

Risk Assessment

Precautionary Principle

\section{ABSTRACT}

In 2015 we reviewed the state of knowledge regarding the potential impact of bisphenol A (BPA) exposure on child neurobehavior. At that time, we expressed concern about the effects of BPA on children's behavior, especially when exposure takes place in utero. Since then, the number of human studies addressing the BPA-neurobehavior hypothesis has doubled, most of them reinforcing previous prenatal associations and frequently showing differences between boys and girls. An increasing number of studies have also shown an association between postnatal BPA exposure and diverse neurobehavioral impairments, including attention-deficit and hyperactivity disorder (ADHD). It may never be possible to establish a causal link between this specific endocrine disruptor and a particular neurobehavioral endpoint; however, research data on the relationship between human BPA exposure and children's behavior has revealed a relatively consistent pattern that cannot be ignored. The mounting experimental and epidemiologic evidence on neurobehavioral effects support more than ever the need to apply the precautionary principle during development, especially in relation to pregnant women and children. It seems that the time to act has arrived.

Neurobehavioral disabilities affect millions of children worldwide, and the prevalence of some neurodevelopmental disorders appears to be increasing. Subclinical symptoms are even more frequent and also contribute to a poorer quality of life and lower academic achievement ${ }^{1}$. Epidemiologic and animal studies have demonstrated that exposure to several families of endocrine-disrupting chemicals (EDCs) contribute to the risk of neurodevelopmental impairment ${ }^{1,2}$.

Bisphenol A (BPA) is a well-known EDC that can interfere with hormonal balance, even at low doses, via multiple steroid hormone receptors that mediate a myriad of cellular effects ${ }^{3}$. The mechanistic understanding of its effects is particularly complex: BPA can bind not only to nuclear and membrane estrogen receptors but also to thyroid, glucocorticoid, and peroxisome proliferator-activated receptors, and it can also interact with steroidogenic enzymes, among other molecular targets ${ }^{4,5}$. This biological promiscuity might explain the pleiotropic effects exerted by BPA on behavior, reproduction, and metabolism ${ }^{6,7}$. The developing brain is a key target for this compound, and pre-, peri- and post-natal BPA exposure has been linked to a variety of altered behaviors, as demonstrated in multiple experimental models ${ }^{3}$.

In 2015 we reviewed the state of knowledge on the relationship between human BPA exposure and neurobehavior ${ }^{5}$. We expressed 
concern about the effects of BPA on children's behavior, especially when exposure takes place in utero. Although only 12 epidemiologic studies were available at that time, their findings pointed in the same direction as experimental studies, suggesting a negative impact of prenatal BPA exposure on children's neurobehavioral functioning in a sex-dependent manner ${ }^{5}$. The results suggested that male fetuses were more frequently affected by prenatal BPA exposure than females, in line with the current hypothesis of environmental intrauterine sex-dependent vulnerability ${ }^{8}$.

Since March 2015, the number of human studies addressing the BPA-neurobehavior hypothesis has doubled $^{9-19}$, most of them reinforcing previous associations and frequently showing differences between boys and girls. For example, earlier findings by Perera et al. (2012) ${ }^{20}$ and Roen et al. (2015) ${ }^{21}$ were confirmed by Perera et al. $(2016)^{13}$, showing a consistent longitudinal pattern of internalizing problems, including anxiety and depression symptoms, among boys from childhood to adolescence in a birth cohort from the U.S.. More recently, Casas et al. $(2015)^{9}$ in Spain, Philippat et al. (2017) $)^{15}$ in France, and Braun et al. (2017) ${ }^{14}$ in Canada all supported the findings of previous studies by Harley et al. $(2013)^{22}$ in California and by Evans et al. (2014) ${ }^{23}$ in a multicenter U.S. cohort. These reported more frequent behavior problems related to prenatal BPA exposure in the males than in the females. Notably, Braun and colleagues $(2017)^{14}$ studied 812 mother-child pairs belonging to the Maternal-Infant Research on Environmental Chemicals (MIREC) study, finding that higher prenatal urinary BPA concentrations were significantly associated with more frequent internalizing problems among three-year-old boys and with poorer executive function and higher social impairments. In contrast, two birth cohorts observed more behavior problems ${ }^{24}$ and social impairments ${ }^{17}$ related to prenatal BPA exposure in girls than in boys. Overall, epidemiologic data support a probable negative effect of prenatal BPA exposure on children's behavior in a sexdependent manner.

An increasing number of studies show that postnatal BPA exposure is also associated with diverse neurobehavioral impairments among both boys and girls ${ }^{10,11,16,21,22}$. For example, some studies, including two representative national surveys, have reported associations with symptoms of attention-deficit and hyperactivity (ADHD) and with the prevalence of ADHD. Findlay and Kohen $(2015)^{10}$ observed positive cross-sectional associations between urinary BPA concentrations and ADHD symptoms in 2730 Canadian children/youths aged 6-17, while Tewar et al. (2016) ${ }^{12}$ found a positive cross-sectional association between BPA concentrations and the risk of ADHD in 460 North American children/youths aged 8-15 years. A more recent case-control study in China also reported a stepwise increase in the risk of ADHD across increasing quartiles of urinary BPA concentrations ${ }^{19}$. These associations reinforce data from some previous prospective studies that found more ADHD symptoms in response to higher prenatal BPA concentrations ${ }^{9,15,24}$. These associations are also supported by experimental data ${ }^{25,26}$.

The human brain is a sexually dimorphic organ, and major morphological differences are permanently shaped during prenatal development under the influence of steroid hormones, especially estrogen and aromatizable androgens ${ }^{27,28}$. BPA has been shown to alter sex-specific structural and behavioral patterns in experimental animals, including non-human primates, increasing, decreasing, and/or eliminating sex differences ${ }^{29-32}$. It is possible that BPA induces these effects through epigenetic modifications related to the estrogen-androgen balance, given evidence that BPA affects the gene expression of several estrogen receptor subtypes (ER $\alpha, E R \beta$ and $E R \gamma$ ) in a sex- and brain region-specific manner ${ }^{29}$. Hence, in utero BPA exposure could predetermine later responses of certain brain areas to steroid hormones. Moreover, a recent high-quality experimental study by the Consortium Linking Academic and Regulatory Insights on BPA Toxicity (CLARITY-BPA) demonstrated that low BPA doses in utero alter the rat brain transcriptome, mainly in the hypothalamus, finding sexspecific effects on hypothalamic ER $\alpha$ and ER $\beta$ expression ${ }^{33}$.

More than $90 \%$ of Europeans and Americans have detectable concentrations of BPA in their urine, and diet is considered the main source of BPA exposure in humans ${ }^{34-37}$. Despite the ubiquity of BPA, its non-persistence and short biological half-life and the episodic nature of its exposure make BPA characterization very difficult, frequently producing an important degree of exposure misclassification. This results in a greater tendency to obtain null results, the so-called attenuation bias ${ }^{15,38}$. Therefore, it is possible that we have been systematically underestimating the effects of BPA, and researchers in the field should consider the potential impact of this attenuation when interpreting epidemiologic findings ${ }^{11,15,38}$. However, despite these methodological limitations, the overall picture of the relationship between human BPA exposure and behavior is relatively consistent across studies and populations. Moreover, the widespread nature of exposure to BPA means that even subtle changes in behavior at the individual level may have relevant effects at the population level, with public health repercussions ${ }^{39,40}$.

In 2008, a National Toxicology Program assessment, based on animal data, reported that BPA-related neural and behavioral endpoints were a major concern for fetuses, infants, and children ${ }^{41}$. Since then, some governments have implemented preventive measures, such as the banning of BPA in baby bottles by Canada and subsequently by the European Union ${ }^{42,43}$, and the total prohibition of BPA 
in France ${ }^{44}$. Additionally, regulatory organizations such as the European Food Safety Authority (EFSA) have been progressively reducing their estimation of the tolerable daily intake (TDI) in subsequent risk assessments. Thus, the TDI for BPA was lowered from $50 \mu \mathrm{g} / \mathrm{kg}$ bw/day to $4 \mu \mathrm{g} / \mathrm{kg} \mathrm{bw} /$ day in 2015 ${ }^{45}$. Nevertheless, recent wellconducted experimental studies have shown that BPA can impact the brain and behavior of rats at doses near to or even below the current TDI ${ }^{33,46}$, and it has been suggested that EFSA's temporary tolerable daily intake of $4 \mu \mathrm{g} / \mathrm{kg}$ bw/day may not be "sufficiently protective" for humans in the general population ${ }^{46}$. EFSA's forthcoming assessment will be an opportunity to integrate new experimental and epidemiologic data and provide evidence in support of action to protect children's behavior.

BPA is commonly found in food packaging materials, being used in the manufacture of polycarbonate plastics and in the epoxy resin liners of food cans. The greater public awareness around BPA has been reflected by the increased popularity of "BPA-free" products ${ }^{47}$. However, many of these products are manufactured using bisphenol analogues, including bisphenol S and F (BPS and BPF), which have been shown to be at least as hormonally active as $\mathrm{BPA}^{48}$. Furthermore, experimental studies of bisphenol substitutes such as BPS and BPF suggest similar and in some cases greater adverse neurobehavioral effects than those associated with $\mathrm{BPA}^{49}$.

It may never be possible to establish a causal link between a specific EDC and a particular neurobehavioral endpoint ${ }^{50}$. Nevertheless, it is impossible to ignore the consistent and accumulating human evidence on the effects of BPA on child neurobehavior. While future research will provide greater clarity, the mounting experimental and epidemiologic data on its neurobehavioral effects support more than ever the need to apply the precautionary principle during development, especially in relation to pregnant women and children ${ }^{5,51}$. It seems that the time to act has arrived ${ }^{52}$.

\section{Conflict of interest}

The authors declare no actual or potential financial conflicts of interest.

\section{Acknowledgements}

This work was supported by research grants from the European Union Commission (H2020-EJP-HBM4EU), the Biomedical Research Networking Center-CIBER de Epidemiología y Salud Pública (CIBERESP). This article will form part of the thesis developed by Vicente Mustieles in the context of the "Clinical Medicine and Public Health Program" of the University of Granada.

\section{References}

1. Grandjean P, Landrigan PJ. Neurobehavioural effects of developmental toxicity. Lancet Neurol. 2014;13(3): 330-338. doi:10.1016/S14744422(13)70278-3.

2. Braun JM. Early-life exposure to EDCs: role in childhood obesity and neurodevelopment. Nat Rev Endocrinol. 2017; 13(3): 161-173. doi:10.1038/nrendo.2016.186.

3. Nesan D, Sewell LC, Kurrasch DM. Opening the black box of endocrine disruption of brain development: Lessons from the characterization of Bisphenol A. Horm Behav. January 2018. doi:10.1016/j. yhbeh.2017.12.001.

4. Wolstenholme JT, Rissman EF, Connelly JJ. The role of Bisphenol A in shaping the brain, epigenome and behavior. Horm Behav. 2011; 59(3): 296-305. doi:10.1016/j.yhbeh.2010.10.001.

5. Mustieles V, Pérez-Lobato R, Olea N, et al. Bisphenol A: Human exposure and neurobehavior. Neurotoxicology. 2015. doi:10.1016/j. neuro.2015.06.002.

6. vom Saal FS, Akingbemi BT, Belcher SM, et al. Chapel Hill bisphenol A expert panel consensus statement: integration of mechanisms, effects in animals and potential to impact human health at current levels of exposure. Reprod Toxicol. 2007; 24(2): 131-138. doi:10.1016/j. reprotox.2007.07.005.

7. Rochester JR. Bisphenol A and human health: a review of the literature. Reprod Toxicol. 2013; 42: 132-155. doi:10.1016/j. reprotox.2013.08.008.

8. DiPietro JA, Voegtline KM. The gestational foundation of sex differences in development and vulnerability. Neuroscience. 2017; 342: 4-20. doi:10.1016/j.neuroscience.2015.07.068.

9. Casas M, Forns J, Martínez D, et al. Exposure to bisphenol A during pregnancy and child neuropsychological development in the INMASabadell cohort. Environ Res. 2015; 142: 671-679. doi:10.1016/j. envres.2015.07.024.

10. Findlay LC, Kohen DE. Bisphenol A and child and youth behaviour: Canadian Health Measures Survey 2007 to 2011. Heal reports. 2015; 26(8): 3-9. http://www.ncbi.nlm.nih.gov/pubmed/26288316. Accessed January 21, 2018.

11. Perez-Lobato R, Mustieles V, Calvente I, et al. Exposure to bisphenol A and behavior in school-age children. Neurotoxicology. 2016; 53: 1219. doi:10.1016/j.neuro.2015.12.001.

12. Tewar S, Auinger P, Braun JM, et al. Association of Bisphenol A exposure and Attention-Deficit/Hyperactivity Disorder in a national sample of U.S. children. Environ Res. 2016; 150: 112-118. doi:10.1016/j. envres.2016.05.040.

13. Perera F, Nolte ELR, Wang Y, et al. Bisphenol A exposure and symptoms of anxiety and depression among inner city children at 10-12 years of age. Environ Res. 2016; 151: 195-202. doi:10.1016/j. envres.2016.07.028.

14. Braun JM, Muckle G, Arbuckle T, et al. Associations of Prenatal Urinary Bisphenol A Concentrations with Child Behaviors and Cognitive Abilities. Environ Health Perspect. 2017; 125(6): 67008. doi:10.1289/ EHP984.

15. Philippat C, Nakiwala D, Calafat AM, et al. Prenatal Exposure to Nonpersistent Endocrine Disruptors and Behavior in Boys at 3 and 5 Years. Environ Health Perspect. 2017; 125(9): 97014. doi:10.1289/ EHP1314.

16. Stacy SL, Papandonatos GD, Calafat AM, et al. Early life bisphenol A exposure and neurobehavior at 8 years of age: Identifying windows of heightened vulnerability. Environ Int. 2017; 107: 258-265. doi:10.1016/j.envint.2017.07.021.

17. Lim YH, Bae S, Kim BN, et al. Prenatal and postnatal bisphenol A exposure and social impairment in 4-year-old children. Environ Heal. 2017; 16(1): 79. doi:10.1186/s12940-017-0289-2.

18. Minatoya M, Araki A, Nakajima S, et al. Cord blood BPA level and child 
neurodevelopment and behavioral problems: The Hokkaido Study on Environment and Children's Health. Sci Total Environ. 2017; 607-608: 351-356. doi:10.1016/J.SCITOTENV.2017.06.060.

19. Li Y, Zhang H, Kuang $H$, et al. Relationship between bisphenol $A$ exposure and attention-deficit/ hyperactivity disorder: A case-control study for primary school children in Guangzhou, China. Environ Pollut. 2018; 235: 141-149. doi:10.1016/j.envpol.2017.12.056.

20. Perera F, Vishnevetsky J, Herbstman JB, et al. Prenatal bisphenol a exposure and child behavior in an inner-city cohort. Environ Health Perspect. 2012; 120(8): 1190-1194. doi:10.1289/ehp.1104492.

21. Roen EL, Wang Y, Calafat AM, et al. Bisphenol A exposure and behavioral problems among inner city children at 7-9 years of age. Environ Res. 2015; 142: 739-745. doi:10.1016/j.envres.2015.01.014.

22. Harley KG, Gunier RB, Kogut K, et al. Prenatal and early childhood bisphenol A concentrations and behavior in school-aged children Environ Res. 2013; 126: 43-50. doi:10.1016/j.envres.2013.06.004.

23. Evans SF, Kobrosly RW, Barrett ES, et al. Prenatal bisphenol A exposure and maternally reported behavior in boys and girls. Neurotoxicology. 2014; 45: 91-99. doi:10.1016/j.neuro.2014.10.003.

24. Braun JM, Kalkbrenner AE, Calafat AM, et al. Impact of Early-Life Bisphenol A Exposure on Behavior and Executive Function in Children. Pediatrics. 2011; 128(5): 873-882. doi:10.1542/peds.2011-1335.

25. Zhou R, Bai Y, Yang R, et al. Abnormal synaptic plasticity in basolateral amygdala may account for hyperactivity and attention-deficit in male rat exposed perinatally to low-dose bisphenol-A. Neuropharmacology. 2011; 60(5): 789-798. doi:10.1016/j.neuropharm.2011.01.031.

26. Ishido M, Masuo Y, Kunimoto M, et al. Bisphenol A causes hyperactivity in the rat concomitantly with impairment of tyrosine hydroxylase immunoreactivity.J Neurosci Res. 2004; 76(3): 423-433. doi:10.1002/ jnr.20050.

27. Bao AM, Swaab DF. Sexual differentiation of the human brain: Relation to gender identity, sexual orientation and neuropsychiatric disorders. Front Neuroendocrinol. 2011; 32(2): 214-226. doi:10.1016/j. yfrne.2011.02.007.

28. Berenbaum SA, Beltz AM. Sexual differentiation of human behavior: Effects of prenatal and pubertal organizational hormones. Front Neuroendocrinol. 2011; 32(2): 183-200. doi:10.1016/j. yfrne.2011.03.001.

29. Kundakovic M, Gudsnuk K, Franks B, et al. Sex-specific epigenetic disruption and behavioral changes following low-dose in utero bisphenol A exposure. Proc Natl Acad Sci. 2013; 110(24): 9956-9961. doi:10.1073/pnas.1214056110.

30. Rubin BS, Lenkowski JR, Schaeberle CM, et al. Evidence of Altered Brain Sexual Differentiation in Mice Exposed Perinatally to Low, Environmentally Relevant Levels of Bisphenol A. Endocrinology. 2006; 147(8): 3681-3691. doi:10.1210/en.2006-0189.

31. Nakagami A, Negishi T, Kawasaki K, et al. Alterations in male infant behaviors towards its mother by prenatal exposure to bisphenol A in cynomolgus monkeys (Macaca fascicularis) during early suckling period. Psychoneuroendocrinology. 2009; 34(8): 1189-1197. doi:10.1016/j.psyneuen.2009.03.005.

32. Negishi T, Nakagami A, Kawasaki K, et al. Altered social interactions in male juvenile cynomolgus monkeys prenatally exposed to bisphenol A Neurotoxicol Teratol. 2014; 44: 46-52. doi:10.1016/j.ntt.2014.05.004

33. Arambula SE, Belcher SM, Planchart A, et al. Impact of Low Dose Oral Exposure to Bisphenol A (BPA) on the Neonatal Rat Hypothalamic and Hippocampal Transcriptome: A CLARITY-BPA Consortium Study. Endocrinology. 2016; 157(10): 3856-3872. doi:10.1210/en.2016-1339.

34. Becker K, Göen T, Seiwert M, et al. GerES IV: phthalate metabolites and bisphenol A in urine of German children. Int J Hyg Environ Health. 2009; 212(6): 685-692. doi:10.1016/j.ijheh.2009.08.002.
35. Calafat AM, Ye X, Wong LY, et al. Exposure of the U.S. population to bisphenol A and 4-tertiary-octylphenol: 2003-2004. Environ Health Perspect. 2008; 116(1): 39-44. doi:10.1289/ehp.10753.

36. Covaci A, Hond E Den, Geens T, et al. Urinary BPA measurements in children and mothers from six European member states: Overall results and determinants of exposure. Environ Res. 2015; 141: 77-85. doi:10.1016/j.envres.2014.08.008.

37. Mustieles V, Ocón-Hernandez O, Mínguez-Alarcón L, et al. Bisphenol A and reproductive hormones and cortisol in peripubertal boys: The INMA-Granada cohort. Sci Total Environ. 2017. doi:10.1016/j. scitotenv.2017.09.093.

38. Perrier F, Giorgis-Allemand L, Slama R, et al. Within-subject Pooling of Biological Samples to Reduce Exposure Misclassification in Biomarker-based Studies. Epidemiology. 2016; 27(3): 378-388. doi:10.1097/EDE.0000000000000460.

39. Bellinger DCDC. What is an adverse effect? A possible resolution of clinical and epidemiological perspectives on neurobehavioral toxicity. Environ Res. 2004; 95(3): 394-405. doi:10.1016/j.envres.2003.07.013.

40. Bellinger DC. Comparing the population neurodevelopmental burdens associated with children's exposures to environmental chemicals and other risk factors. Neurotoxicology. 2012; 33(4): 641643. doi:10.1016/j.neuro.2012.04.003.

41. Chapin RE, Adams J, Boekelheide K, et al. NTP-CERHR expert panel report on the reproductive and developmental toxicity of bisphenol A. Birth Defects Res B Dev Reprod Toxicol. 2008; 83(3): 157-395. doi:10.1002/bdrb.20147.

42. Canada Consumer Product Safety Act. 2017. http://laws-lois.justice. gc.ca/PDF/C-1.68.pdf. Accessed January 16, 2018.

43. European Commission - Bisphenol A: EU ban on baby bottles to enter into force tomorrow. http://europa.eu/rapid/press-release_IP-11664_en.htm. Published 2011. Accessed January 16, 2018.

44. Audran X, Salmon DG. French Law Banning Bisphenol A in Food Containers Enacted BPA Ban in Food Containers by 2015. 2012. https://gain.fas.usda.gov/Recent GAIN Publications/French Law Banning Bisphenol A in Food Containers Enacted_Paris France_2-5-2013.pdf. Accessed January 16, 2018.

45. Scientific Opinion on the risks to public health related to the presence of bisphenol A (BPA) in foodstuffs. EFSA J. 2015; 13(1): 3978. doi:10.2903/j.efsa.2015.3978.

46. Hass U, Christiansen S, Boberg J, et al. Low-dose effect of developmental bisphenol A exposure on sperm count and behaviour in rats. Andrology. 2016; 4(4): 594-607. doi:10.1111/andr.12176.

47. Vandenberg LN, Prins GS. Clarity in the face of confusion: new studies tip the scales on bisphenol A (BPA). Andrology. 2016; 4(4): 561-564. doi:10.1111/andr.12219.

48. Rochester JR, Bolden AL. Bisphenol S and F: A Systematic Review and Comparison of the Hormonal Activity of Bisphenol A Substitutes. Environ Health Perspect. 2015; 123(7): 643-650. doi:10.1289/ ehp.1408989.

49. Rosenfeld CS. Neuroendocrine disruption in animal models due to exposure to bisphenol A analogues. Front Neuroendocrinol. 2017; 47: 123-133. doi:10.1016/j.yfrne.2017.08.001.

50. Colborn T. Neurodevelopment and endocrine disruption. Environ Health Perspect. 2004; 112(9): 944-949. http://www.ncbi.nlm.nih. gov/pubmed/15198913. Accessed January 22, 2018.

51. Vandenberg LN. Exposure to bisphenol A in Canada: invoking the precautionary principle. Can Med Assoc J. 2011; 183(11): 1265-1270. doi:10.1503/cmaj.101408.

52. Fernández MF, Román M, Arrebola JP, et al. Endocrine Disruptors: Time to Act. Curr Environ Heal Reports. 2014; 1(4): 325-332. doi:10.1007/ s40572-014-0025-9. 